\title{
THE NEON NOVA. III. THE INFRARED LIGHT CURVES OF NOVA QU VULPECULAE (NOVA VUL 1984 \#2)
}

\section{R. D. GEHRZ AND T. J. JONES}

Department of Astronomy, School of Physics and Astronomy, 116 Church Street, S. E., University of Minnesota, Minneapolis, Minnesota 55455

Electronic mail: gehrz@ast1.spa.umn.edu

K. MATtheWS AND G. NeUgebauer

Palomar Observatory, California Institute of Technology, 320-47, Pasadena, California 91125

\section{Charles E. WOODWARD ${ }^{1}$}

Wyoming Infrared Observatory, Department of Physics and Astronomy, University of Wyoming, Laramie, Wyoming 82071-3905 Electronic mail: chelsea@wapiti.uwyo.edu

\section{T. L. HAYWARD}

Center for Radiophysics and Space Research, Cornell University, Ithaca, New York 14853

\section{A. GREENHOUSE}

Laboratory for Astrophysics, National Air and Space Museum, Smithsonian Institution, Washington DC 20260

Electronic mail: matt@wright.nasm.edu

Received 1994 December 13; revised 1995 March 28

\begin{abstract}
We report 1.25 to $19.5 \mu \mathrm{m}$ broadband infrared (IR) photometric measurements acquired during an eight year period on the prototypical ONeMg "neon nova" QU Vulpeculae (Nova Vul 1984 \#2). The energy distribution of the ejecta evolved through several phases. An early free-free emission phase was followed by an IR coronal phase characterized by the appearance of strong emission lines from forbidden atomic transitions. The lines of [ $\mathrm{Ne} \mathrm{VI}]$ at $7.6 \mu \mathrm{m}$, and [Ne II] at $12.8 \mu \mathrm{m}$ were especially strong during the coronal phase. A small amount of silicate dust condensed in the ejecta after about a year. The evidence provided by our IR observations for high abundances of metals in the ejecta of QU Vul is reviewed. We present the IR light curves of QU Vul, and show that the temporal development of its persistent IR coronal emission phase was evident in the broadband $K(2.3 \mu \mathrm{m})$ and $L(3.6 \mu \mathrm{m})$ photometry. Using data from our previous studies of classical novae, we suggest that $K$ and $L$ photometry can distinguish between the slower ONeMg novae with persistent IR coronal activity and CO novae that produce copious quantities of circumstellar dust. The most striking signature is produced in the $L$ band, which contains emission from [Mg VIII] at $3.02 \mu \mathrm{m}$, [Al VI] at $3.66 \mu \mathrm{m}$, and [Si IX] at $3.92 \mu \mathrm{m}$. We comment on the peculiar tendency of the IR light curves of novae to decay exponentially. (C) 1995 American Astronomical Society.
\end{abstract}

\section{INTRODUCTION}

QU Vul (Nova Vulpeculae 1984 \#2), the archetypal "neon nova," provides the paradigm for infrared (IR) studies of the physical characteristics of a thermonuclear runaway (TNR) on an oxygen-neon-magnesium (ONeMg) white dwarf (WD) in a close binary system. IR observations of QU Vul by Gehrz et al. (1985, Paper I) 140 days after its eruption revealed the strongest $12.8 \mu \mathrm{m}$ [Ne II] forbidden emission line relative to the continuum ever observed in an astrophysical source. Their observations were the first to confirm a theoretical prediction by Ferland \& Shields (1978) that [Ne II] emission can be a significant source of cooling in the circumstellar shells of classical novae. About $80 L_{\odot}$ was radiated in the $12.8 \mu \mathrm{m}$ line 141 days after the outburst. This was $0.1 \%$ of the outburst luminosity and nearly $1 \%$ of the

${ }^{1}$ Presidential Faculty Fellow. total shell luminosity. Subsequent IR observations of QU Vul by our group revealed 10 and $20 \mu \mathrm{m}$ emission features 240 days after the outburst demonstrating that a small amount of dust composed of oxygen rich silicate grains had condensed in the ejecta (Gehrz et al. 1986, Paper II). A year after the outburst, QU Vul entered a persistent IR coronal phase characterized by a thermal bremmstrahlung continuum with strong emission from IR forbidden fine structure lines (see Greenhouse et al. 1988, 1990) of members of the $B$ and $F$ isoelectronic sequences including $[\mathrm{Ne} \mathrm{VI}]$ at $7.63 \mu \mathrm{m}$, [Mg VII] at $3.02 \mu \mathrm{m}$, [Si VII] at $2.47 \mu \mathrm{m}$, [Si IX] at $3.92 \mu \mathrm{m}$, [Al IX] at $2.04 \mu \mathrm{m}$, and [Al vI] at $3.66 \mu \mathrm{m}$. The [Mg VIII] and $[\mathrm{Al} \mathrm{VI}]$ lines were by far the strongest contributors to the emission in the $L$ broadband. We presented detailed quantitative arguments leading to the conclusion that $\mathrm{Ne}, \mathrm{Mg}, \mathrm{Si}$, and $\mathrm{Al}$ were probably substantially overabundant in QU Vul (Papers I and II; Greenhouse et al. 1990). A recent analysis 
of optical and IUE spectra by Saizar et al. (1992) substantiates this conclusion.

The observations described above tend to confirm the existence of $\mathrm{ONeMg}$ WDs in binary star systems capable of producing nova explosions, and we hereafter refer to them as ONeMg, or "neon" novae (see Gehrz et al. 1993 for a review of the characteristics of these systems). Such novae may be capable of injecting significant quantities of peculiar isotopes such as ${ }^{13} \mathrm{C},{ }^{15} \mathrm{~N},{ }^{22} \mathrm{Na}$, and ${ }^{26} \mathrm{Al}$ into the interstellar medium (ISM) on local scales. V1974 Cyg has recently been shown by Hayward et al. (1992) to be another nova with IR characteristics similar to those of QU Vul. The IR spectral energy distributions (SEDs) of QU Vul and V1974 Cyg can generally be characterized as showing persistent strong forbidden line radiation and little or no thermal emission from dust.

Classical novae of the DQ Hercules class form optically thick (at $V=0.55 \mu \mathrm{m}$ ) circumstellar dust shells composed largely of carbon grains, and their IR SEDs are dominated by strong thermal emission from the dust. Recent examples are FH Ser (Hyland \& Neugebauer 1970; Geisel et al. 1970), NQ Vul (Ney \& Hatfield 1978), LW Ser 1978 (Gehrz et al. 1980a), and V705 Cas (Gehrz 1995). These dust formation events appear to occur in binary systems where the primary is a carbon-oxygen (CO) WD, and we refer to them henceforth as CO novae. Optically thick dust shells usually reach their maximum visual optical depth 50-80 days after outburst when they reradiate the maximum outburst luminosity in the IR. Occasionally, a smaller amount of dust forms in faster $\mathrm{CO}$ novae. In these cases there is IR emission from the dust, but there is no deep visual minimum due to dust extinction as there is in the DQ Her novae. The prime example of this behavior was the moderately fast CO nova V1668 Cyg which condensed an optically thin carbon dust shell with a maximum visual optical depth of $\approx 0.1$ about 60 days after outburst (Gehrz et al. 1980b). A cardinal characteristic of the IR light curves of the CO novae is that they show a rapid, large-amplitude rise as the dust forms followed by a steep decline as the dust formation process subsides.

A handful of novae defy unambiguous classification into the $\mathrm{CO}$ or ONeMg classes based upon their IR behavior. For example, the very fast ONeMg nova V838 Her condensed a dust shell with a maximum visual optical depth of 0.05 in 25 days (Woodward et al. 1992). V1370 Aql condensed a thick dust shell containing several types of grains (Williams \& Longmore 1984; Bode et al. 1984; Gehrz et al. 1984), but showed spectral evidence of being an ONeMg nova (private communication by S. G. Starrfield). V827 Her (Her 1987) had a fairly short-lived IR coronal phase (Greenhouse et al. 1990) and also formed a dust shell with an optical depth of about 0.12 (Gehrz 1988a). V1500 Cyg, possibly a very fast $\mathrm{ONeMg}$ nova judging from the abundances reported for it by Lance et al. (1988), showed a very brief IR coronal emission phase (Grasdalen \& Joyce 1976).

The wide range of IR dust formation behaviors and coronal emission scenarios exhibited by novae led us to initiate an expanded program to monitor their IR temporal development. Here we present a photometric record of the IR temporal development of the prototypical neon nova QU Vul covering a time period of more than eight years following its eruption. We discuss the morphology of the light curves of QU Vul, and compare the characteristics of the near-IR light curves of the ONeMg novae with persistent IR coronal emission and CO novae that form thick dust shells.

\section{OBSERVATIONS}

The IR temporal development of QU Vul was monitored from 1984 March 17 UT to 1992 September 19 UT by the IR groups at the University of Minnesota (UM), the University of Wyoming (UW), and the California Institute of Technology (CIT). Table 1 and Figs. 1 and 2 summarize our entire photometric data record for this period, which covers a time span of more than 2800 days from the time of the eruption on day $0=1984$ Dec 24.5 UT. Statistical uncertainties are given when they exceed 0.05 magnitudes. The UM/UW data taken through 28 August 1986 UT, previously reported piecemeal in Papers I and II, and by Greenhouse et al. (1988), are collected in Table 1 for completeness of the photometric record. We have also included in Table 1 several very early IR measurements reported by British (Burton et al. 1984) and Russian (Bergner et al. 1984) observers in IAU Circulars No. 4024 and 4033.

The UW photometry was obtained using a Wyoming multifilter $1.2 \mathrm{~K} \mathrm{GaGe}$ bolometer with a $5^{\prime \prime}$ beam operated at the $f / 27$ Cassegrain focus of the $2.34 \mathrm{~m}$ Wyoming Infrared Telescope (Gehrz \& Hackwell 1978). Bandpasses and calibration for the Wyoming photometer are given in Gehrz et al. (1974) and Gehrz et al. (1987). UM measurements were made using a Minnesota multifilter $1.2 \mathrm{~K} \mathrm{GaGe}$ bolometer photometer with a $26^{\prime \prime}$ diaphragm on the $0.7 \mathrm{~m}$ University of Minnesota 0'Brien telescope. Ney \& Hatfield (1978) have described the Minnesota photometric system.

The Caltech near-IR observations were obtained at the $f / 70$ Cassegrain focus of the 200 in. Hale Telescope using a single element InSb detector. The observations were taken with a $5^{\prime \prime}$ diaphragm through filters defining the near-IR photometric bands $J=1.27 \mu \mathrm{m}, H=1.65 \mu \mathrm{m}, K=2.2 \mu \mathrm{m}$, and $L^{\prime}=3.7 \mu \mathrm{m}$. The emission of the sky was subtracted by "chopping" the secondary mirror of the telescope at a frequency of $5 \mathrm{~Hz}$ and an angular distance of $15^{\prime \prime}$ North/South. Photometric calibration was maintained through observations of the standard stars of Elias et al. (1982). The Caltech $N$-band observations were obtained using a 4.6 " beam with a standard Infrared Laboratories $\mathrm{GaGe}$ bolometer at the $f / 70$ Cassegrain focus of the 200 in. telescope; these measurements were made and calibrated as described by Gehrz et al. (1980a).

Although the three photometric systems used for our measurements have slightly different effective wavelengths, we have for simplicity referred all measurements to the Wyoming system. The color corrections required to compare the data taken on the different systems for the purposes of the analysis that follows are generally negligible compared to the photometric uncertainties, and no corrections have been applied in the production of Figs. 1 and 2 or the interpretation that follows. These systems have been used together 


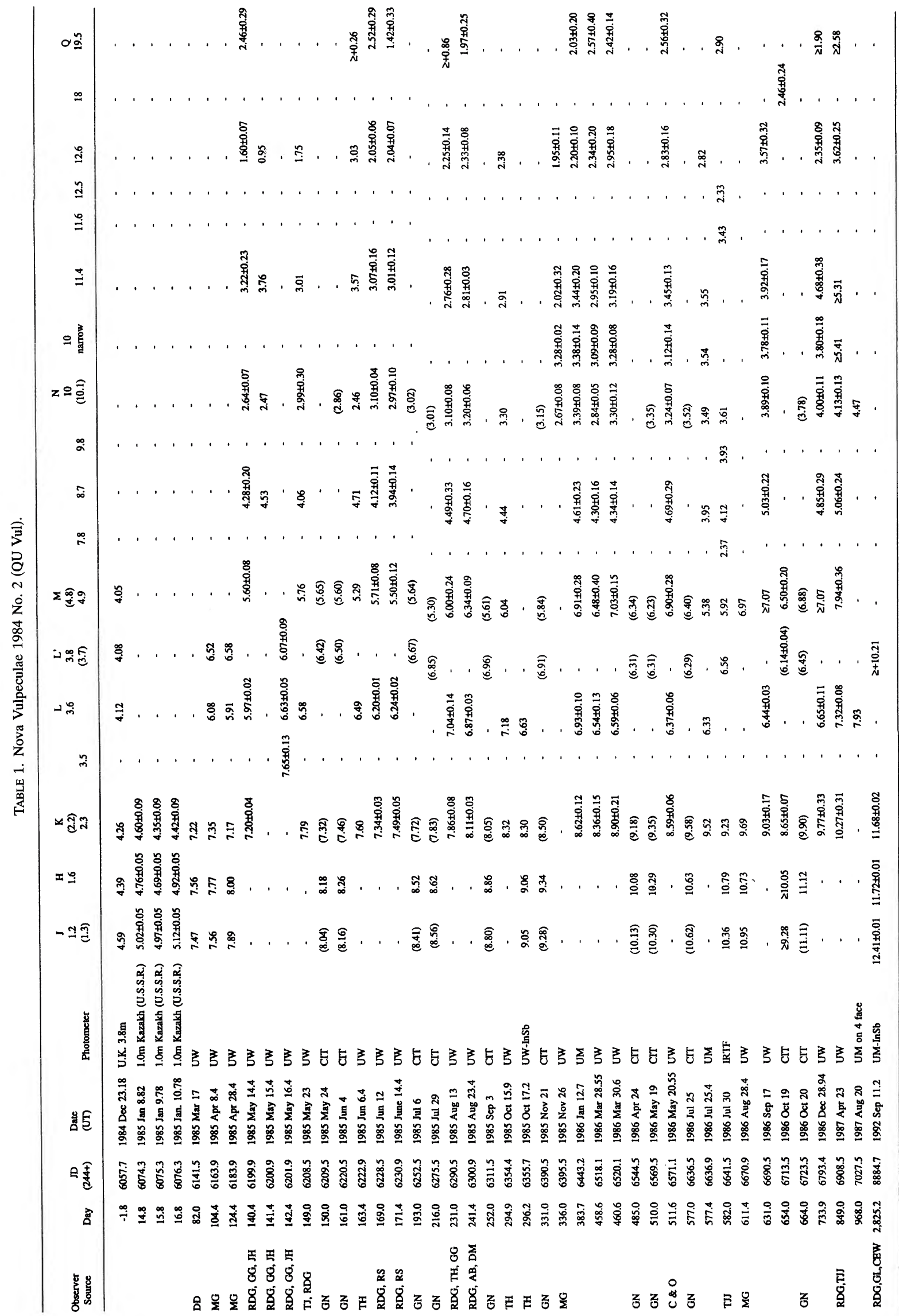



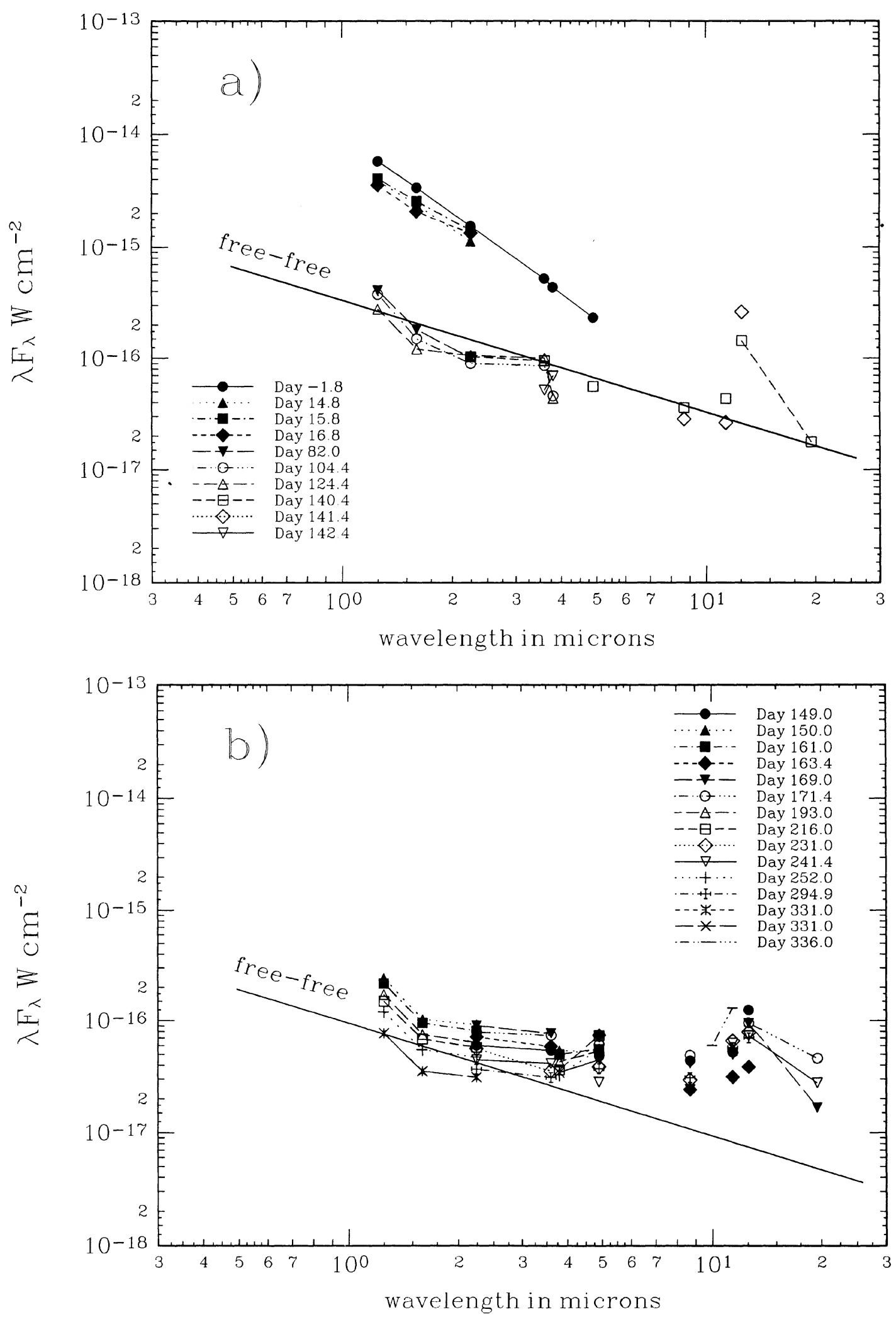

FIG. 1. IR energy distribution of QU Vul at several epochs showing the transition from the free-free emission phase to the coronal phase. Panel (a) shows the early free-free phase, and the onset of the $12.8 \mu \mathrm{m}$ [Ne II] emission phase preceding the appearance of strong near-IR coronal emission. In panel (b), weak near-IR coronal emission and silicate dust emission at 7-14 $\mu \mathrm{m}$ have flattened out the energy distribution. Panel (c) shows the energy distribution during the persistent near-IR coronal phase that lasted from 1 to at least $3 \mathrm{yr}$ after the eruption. The data for day 2825 show that weak IR coronal lines were still present eight years after the outburst. 


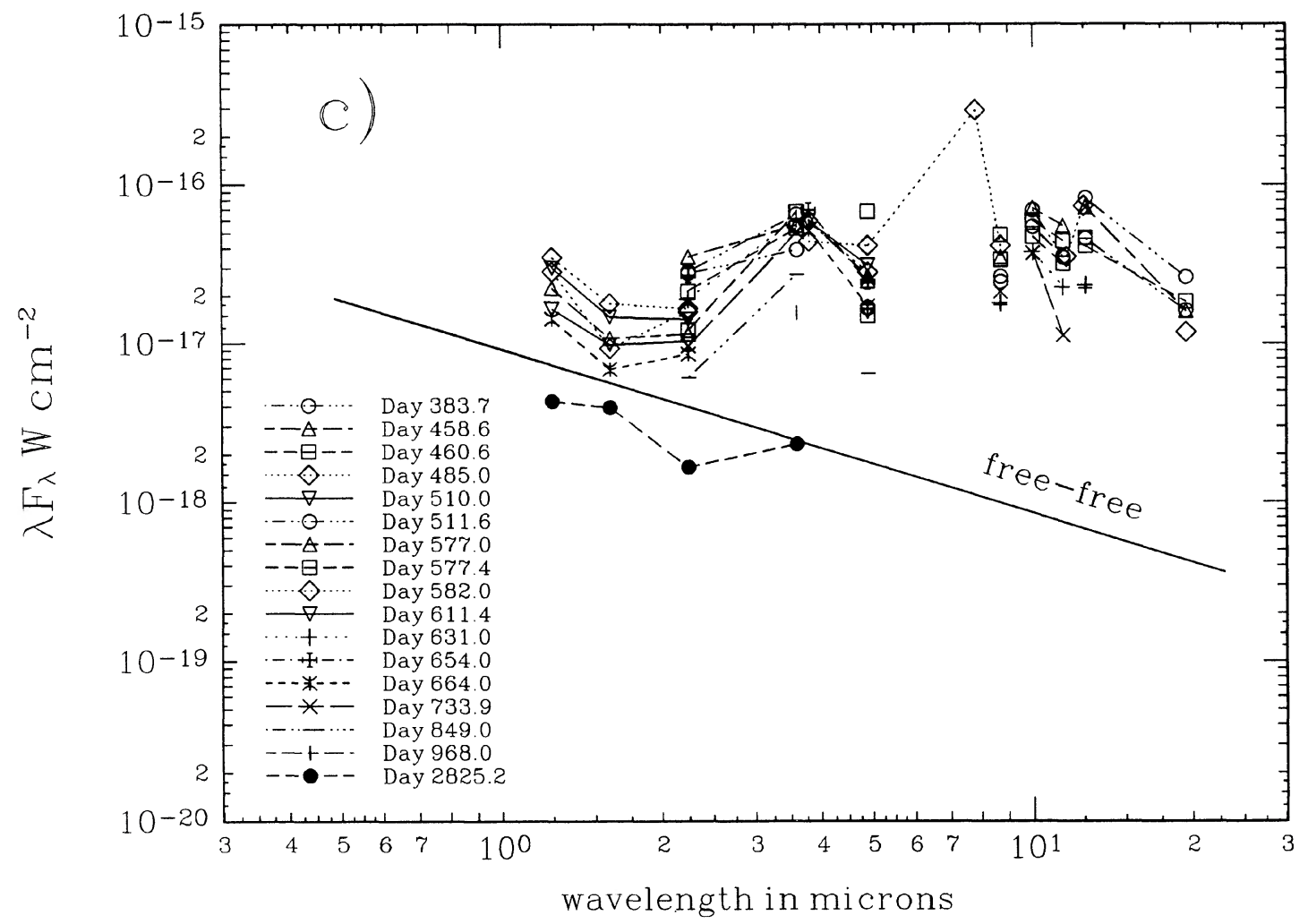

FIG. 1. (continued)

previously in describing the IR temporal development of LW Ser (Gehrz et al. 1980a), V1668 Cyg (Gehrz et al. 1980b), and PW Vul (Gehrz et al. 1988).

\section{DISCUSSION}

Figures 1(a)-1(c) show the spectral energy distributions (SEDs) of the major phases that we identified during the eight years of QU Vul's post-eruption evolution. We present the IR light curves of QU Vul in Figs. 2(a)-2(1), the $V$ light curve in Fig. 3, and a comparison of QU Vul's $K$ and $L$ light curves with those of other recent novae in Figs. 4(a) and 4(c). The onset of the eruption (Day 0) was estimated to be $\approx$ JD 244,6059.5 (1984 December 24.5 UT) from the $V$ maximum using photometric data available in the IAU Circulars and the AAVSO data shown in Fig. 3 (Mattei 1994). We adopt this date as the onset of the shell ejection in the discussion that follows. Table 2 summarizes other important physical parameters describing the outburst of QU Vul that can be calculated from our data and the data obtained by other investigators.

\subsection{Temporal Development of the Spectral Energy Distribution}

The IR SED of QU Vul's ejecta, as defined by broadband IR filters, evolved through two phases following the initial fireball expansion. These are shown in Figs. 1(a)-(c). An early free-free emission phase [Fig. 1(a)] was similar to that exhibited by all other classical novae (see Ney \& Hatfield 1978; Gehrz 1988, 1990) with the exception that excitation conditions were high enough by day 140 that strong $12.8 \mu \mathrm{m}$ [Ne II] emission was present. This feature was also observed during the free-free emission phase of the ONeMg nova V1974 Cyg (Hayward et al. 1992). The presence of the line is manifested by the large excess in the $12.6 \mu \mathrm{m}$ filter as compared to the continuum defined by the other photometric points.

As excitation continued to increase from day 150 to day 336 [Fig. 1(b)], the SED flattened to $F_{\nu} \approx$ const as increasing near-IR coronal emission began to dominate the $K$ and $L$ bands, and as silicate dust emission became pronounced in the 7-14 $\mu \mathrm{m}(N)$ and $Q$ bands after day 240 (Paper II). The development of the silicate dust emission features is tracked primarily in the excesses over the continuum that appear in the $10,11.4$, and $19.5(Q) \mu \mathrm{m}$ filters. IR spectroscopy (Paper II) confirmed that the silicate emission in the 10 and $11.4 \mu \mathrm{m}$ filters was not contaminated by strong line emission.

The epoch from day 384 to day 968 [Fig. 1(c)] marks the strong IR coronal phase in which the free-free continuum had superimposed emission lines from near-IR forbidden atomic transitions of members of the $B$ and $F$ isoelectronic sequences. Strong emission from [Ne VI] at $7.6 \mu \mathrm{m}$, and decreased [Ne II] emission at $12.8 \mu \mathrm{m}$ also characterized the strong IR coronal phase. These line ratios are also consistent with an increase in the excitation temperature of the central source. Greenhouse et al. $(1988,1990)$ have argued that the 

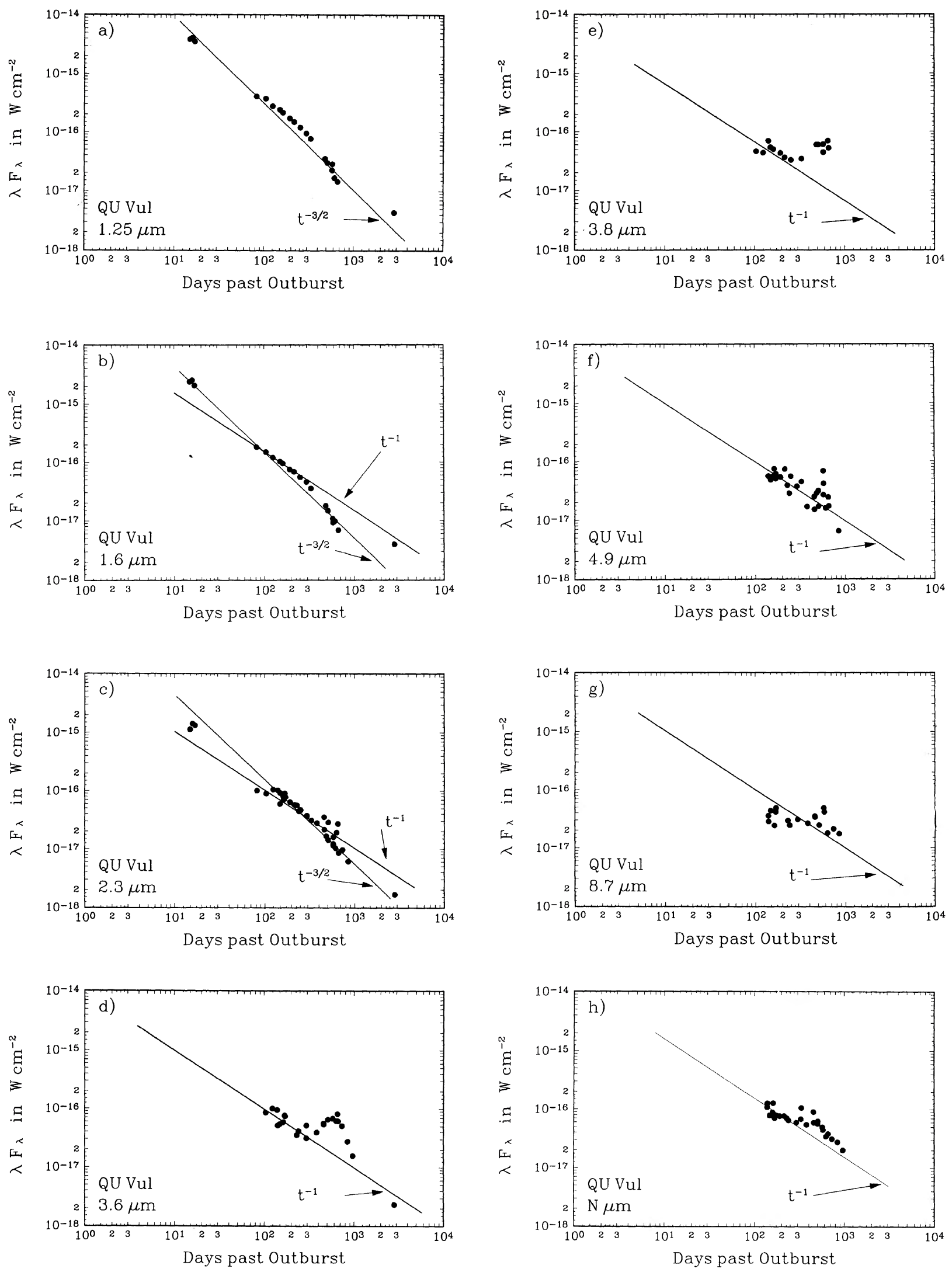

FIG. 2. Panels (a)-(1) show the IR light curves of QU Vul. It is clear that the $t^{-2}$ and $t^{-3}$ decline rates that characterize the temporal evolution of the light curves of some novae do not apply in the case of QU Vul. The $3.6 \mu \mathrm{m}$ light curve shows a pronounced secondary "maximum" during the IR coronal phase from day 100 to about day 800 . 

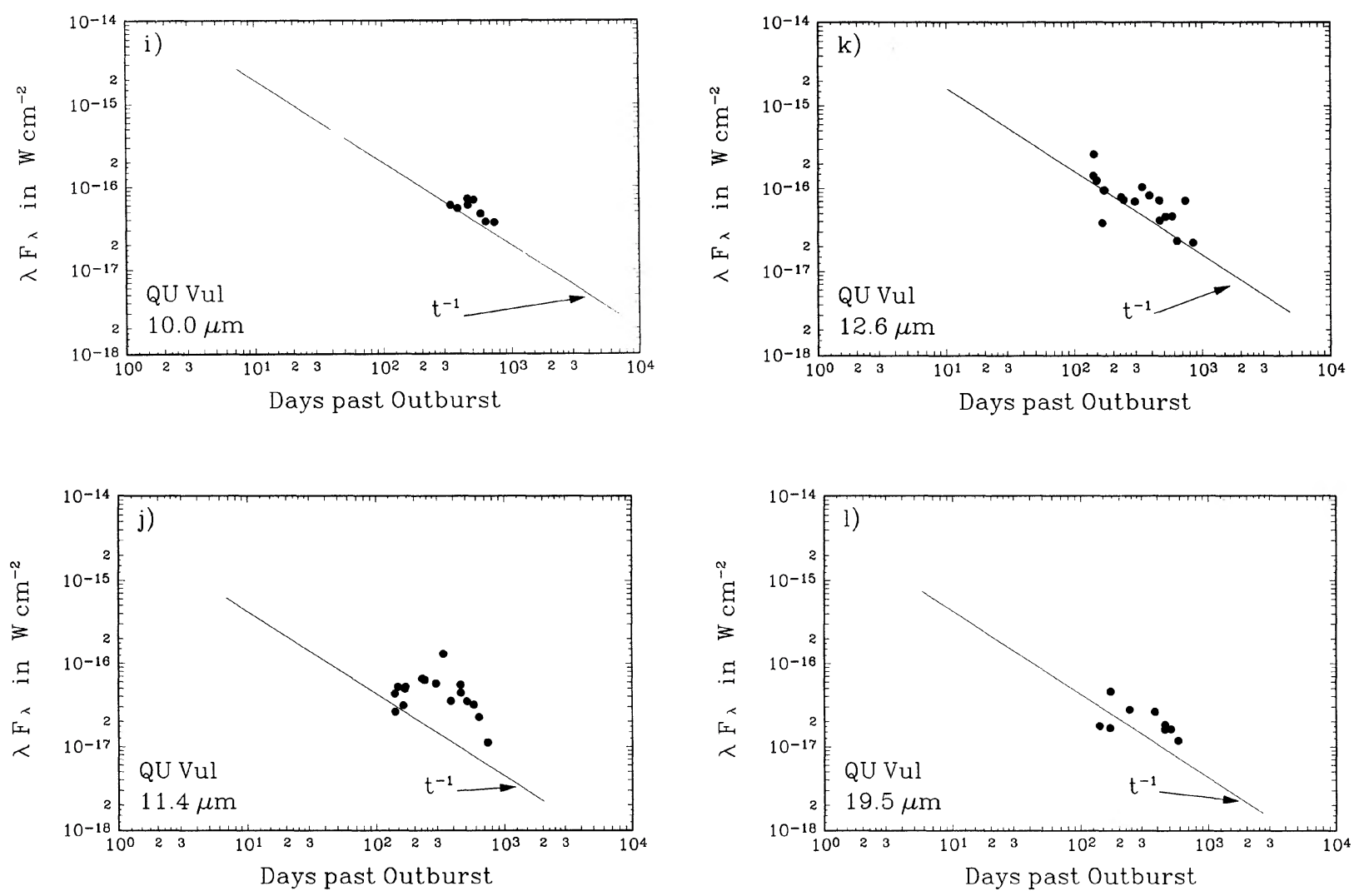

Fig. 2. (continued)

emission line structure during this phase requires the temperature of the central engine of the nova to have been nearly $500,000 \mathrm{~K}$. EXOSAT observations of soft $\mathrm{X}$ rays from QU Vul by Ögelman et al. (1987) suggest that the central en- gine's temperature had already risen as high as 250,000 $300,000 \mathrm{~K}$ by 307 days after the outburst. Our data [Fig. 1(c)] show that this high-temperature central engine must have persisted during the period from 1 to at least 3 yr fol-

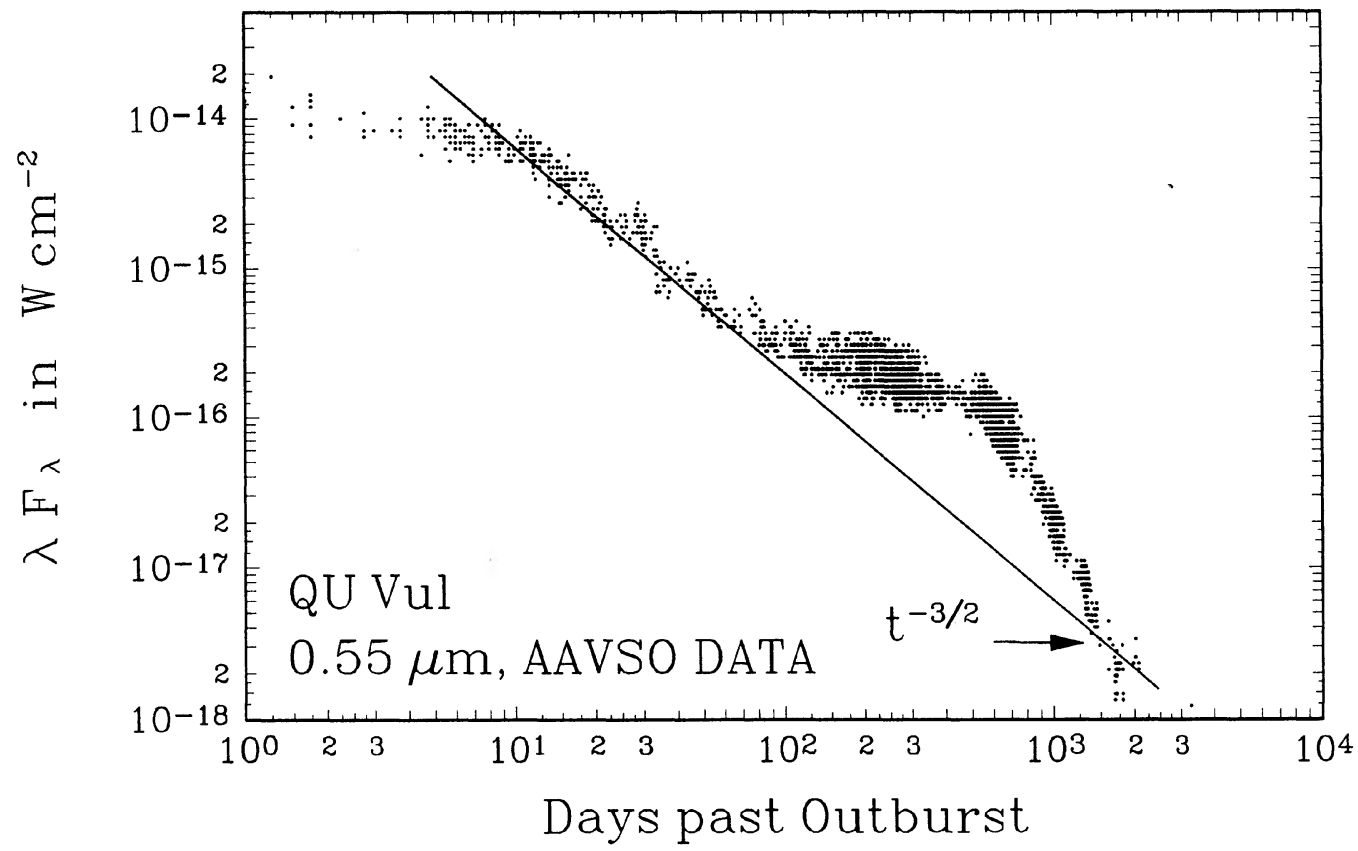

FIG. 3. The visual light curve of QU Vul. Data are from The American Association of Variable Star Observers (Mattei 1994). Like the 3.6 $\mu$ m light curve [Fig. 2(d)], the $V$ light curve shows a pronounced secondary "maximum" during the IR coronal phase from day 100 to about day 800 . 

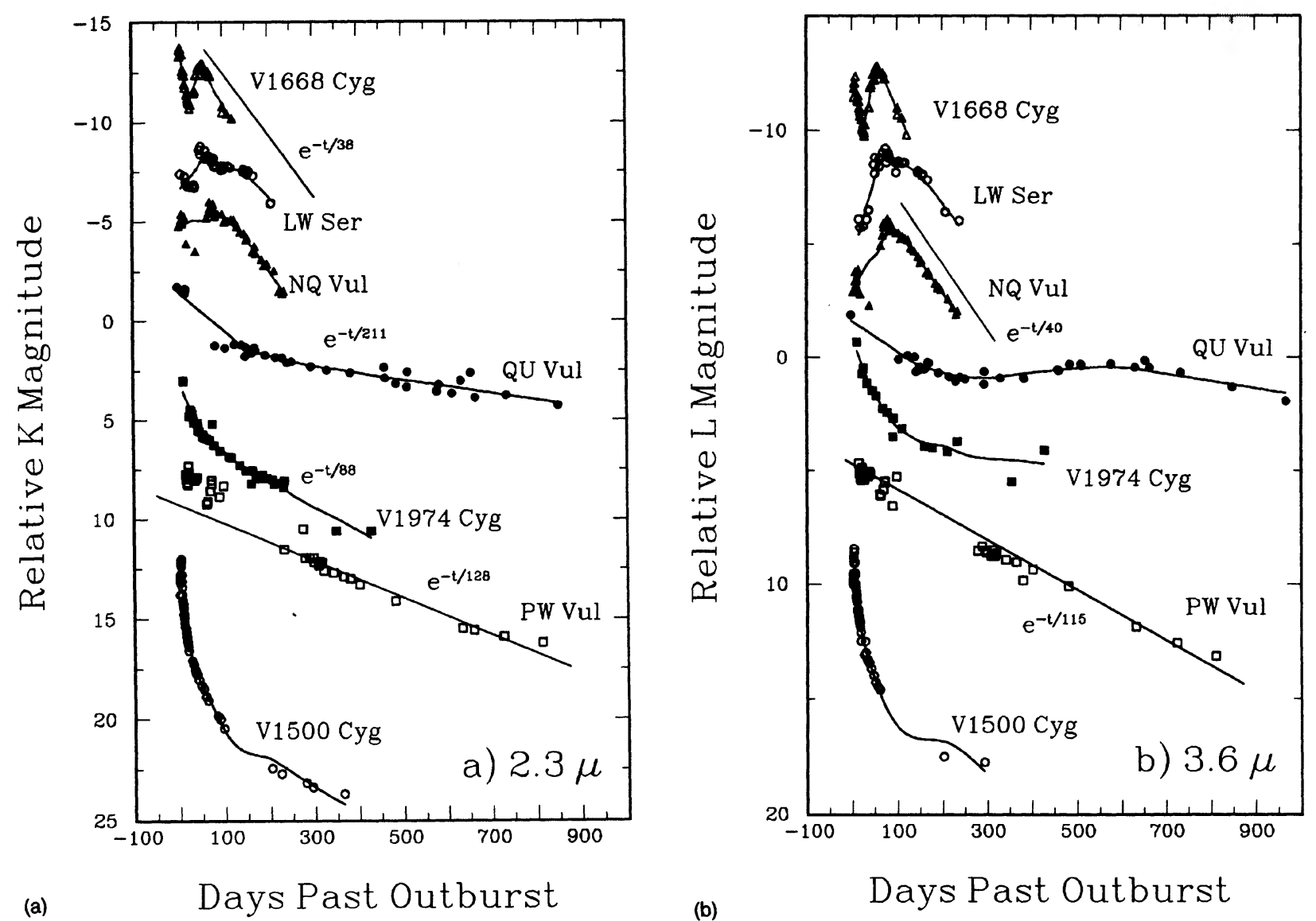

FIG. 4. The $K$ [panel (a)] and $L$ [panel (b)] light curves of seven classical novae showing that the ONeMg novae QU Vul (data from this paper) and V1974 Cyg (data from Woodward et al. 1995b) are particularly distinguished by elevated emission in the $L$ band during their persistent IR coronal phases. The CO novae with optically thick (at $V$ ) dust shells LW Ser (data from Gehrz et al. 1980a) and NQ Vul (data from Ney \& Hatfield 1978) peak early during the dust formation phase and decline exponentially thereafter. The CO nova V1668 Cyg (data from Gehrz et al. 1980b) formed an optically thin (at $V$ ) dust shell and had $K$ and $L$ light curves similar to those of the optically thick CO dust shells. V1500 Cyg (data from Gallagher \& Ney 1976; Ennis et al. 1977) was a fast nova that showed neither persistent IR coronal emission nor dust formation. PW Vul (data from Gehrz et al. 1988a) was a slower nova with neither persistent IR coronal nor dust emission that decayed exponentially. Note the peculiar tendency of nova IR light curves to decay exponentially over rather long time scales at some epochs. The mean lifetime ( $e$-folding time) for the decline rate in a given nova appears to vary from wavelength to wavelength.

lowing the eruption. The excitation appears to have decreased again by day 2825 as evidenced by the decline in the excess fluxes in the $K$ and $L$ bands [see also Figs. 4(a) and 4(b)] caused by near-IR coronal emission and by a recent reappearance of the $12.8 \mu \mathrm{m}$ emission line (Gehrz et al., to be published). The IR coronal phase SEDs for the ONeMg QU Vul and V1974 Cyg, both of which showed strong and persistent IR coronal line emission, do not conform well to the blackbody shape exhibited by dust-forming novae. Instead, the shape of the SED is primarily determined by excess radiation in the various broad- and narrow band filters due to line emission. We conclude that the IR coronal phase in $\mathrm{ONeMg}$ novae can be readily distinguished from the dust formation phase in very dusty $\mathrm{CO}$ novae by inspection of broadband IR SEDs such as those shown in Figs. 1(a)-1(c). The SEDs we present here for QU Vul should prove useful as templates for identifying ONeMg novae with persistent IR coronal emission in the distant reaches of the galaxy and in other galaxies by sensitive space platforms like the European
Space Agency's Infrared Space Observatory (ISO) and NASA's Space Infrared Telescope Facility (SIRTF).

\subsection{The Infrared Light Curves of $Q U$ Vul}

The IR light curves of QU Vul are shown in Figs. 2(a)2(1). Figure 3 shows the visual light curve for comparison. A general examination of the temporal characteristics of $Q U$ Vul's light curves, and comparison of the curves with those of other novae [see Figs. 4(a) and 4(c)] leads to several conclusions.

(1) The light decline rates for QU Vul are substantially slower than are those for neon novae like V1500 Cyg that exhibit little or no dust formation and short-lived near-IR coronal emission phases. The IR SEDs of novae like V1500 Cyg are dominated for the most part by pure free-free emission with relatively weak overlying emission lines. In such cases, the light decline rates begin with a $t^{-2}$ dependence as the ejected shell expands with uniform thickness and in- 
TABLE 2. Summary of physical properties of QU Vul.

\begin{tabular}{|c|c|c|}
\hline Parameter & $\begin{array}{c}\text { Relationship or } \\
\text { Source }\end{array}$ & Value for $Q V$ Vul \\
\hline RA (1950) & Klemola 1984 & $20^{\mathrm{h}} 24^{\mathrm{m}} 40^{\mathrm{s}} \cdot 53$ \\
\hline DEC $(1950)$ & Klemola 1984 & $+27^{\circ} 40^{\prime} 48.2^{\prime \prime}$ \\
\hline Day 0 & visual maximum & JD $2,446,059.5$ \\
\hline $\mathrm{v}_{\mathrm{o}}$ & IAU Circ. 4184 & \\
\hline & Paper I & $1000-2500 \mathrm{~km} \mathrm{sec}-1$ \\
\hline$\left(m_{v}\right)_{\max }$ & Paper I & +5.7 \\
\hline $\mathbf{A}_{\mathbf{v}}$ & Paper I & $\approx 1.0 \mathrm{mag}$ \\
\hline$t_{2}, t_{3}$ & $\begin{array}{l}\text { Gehrz et al. } 1988 \mathrm{a}, \\
\text { and AAVso data }\end{array}$ & 27,40 days \\
\hline $\mathbf{M}_{\mathrm{v}}$ & $t_{2}$ and $t_{3}$ & $-7.5 \pm 0.2$ \\
\hline $\mathbf{M}_{\mathrm{bol}}$ & B.C. $=-0.1$ & $-7.6 \pm 0.2$ \\
\hline $\mathrm{D}_{2,3}$ & distance modulus & $3 \mathrm{kpc}$ \\
\hline$\left(\lambda F_{\lambda}\right)_{\max }$ & $\begin{array}{l}m_{v} \text { at maximum light } \\
\text { and } A_{v} \approx 1 \text { mag }\end{array}$ & $3 \times 10^{-14} \mathrm{~W} \mathrm{~cm}^{-2}$ \\
\hline$\left(\lambda F_{\lambda}\right)_{\max }$ & IR from ejecta & $\approx 10^{-15} \mathrm{~W} \mathrm{~cm}-2$ \\
\hline $\mathrm{L}_{\mathrm{o}}$ & $\mathrm{M}_{\mathrm{v}}$ and $\mathrm{BC}=-0.1$ & $10^{5} \mathrm{~L}_{\odot} \cdot$ \\
\hline$\left(L_{O}\right)_{I R}$ & $\left(\lambda F_{\lambda}\right)_{\max }$ in $I R, D$ & $\approx 8 \times 10^{4} \mathrm{~L}_{\odot}$ \\
\hline$\tau_{10}$ & $\begin{array}{l}\text { silicate optical depth, } \\
\text { Paper II }\end{array}$ & $4 \times 10^{-3}$ \\
\hline$M_{\text {dust }}$ & $\tau_{10}$, shell radius & $1.5 \times 10^{-7} \mathrm{M}_{\odot}$ \\
\hline
\end{tabular}

creases to $t^{-3}$ as sonic disturbances drive the shell into free expansion (see Ennis et al. 1977). We interpret the slow decline rate of some of the broadband light curves of QU Vul as being caused by increasingly strong forbidden line emission due to the steadily increasing temperature of the central engine. As would be expected for this scenario, the light decline rates are lowest for the filters most affected by line emission like $K, L, L^{\prime}(3.8 \mu \mathrm{m})$ and $12.6 \mu \mathrm{m}$. Other filters such as $10 \mu \mathrm{m}, 11.4 \mu \mathrm{m}$, and $Q(19.5 \mu \mathrm{m})$ are affected by silicate dust emission.

(2) It is apparent from Figs. 2(a)-2(1) that the persistent IR coronal phase of QU Vul between days 200 and 1000 is particularly well characterized by the increases over the baseline decay rate in the $V$ (Fig. 3), $L$ [Fig. 2(d)], and $L^{\prime}$ [Fig. 2(e)] light curves. In fact, $L$-band or $L$-prime photometry alone would be a good diagnostic indicator for studies of novae in distant galaxies using ISO or SIRTF. The $1.2 \mu \mathrm{m}$ wide $L$ band contains two of the strongest IR coronal lines known in ONeMg novae: [Mg VIII] at $3.02 \mu \mathrm{m}$ and [Al VI] at $3.66 \mu \mathrm{m}$. The weaker [Si IX] line at $3.92 \mu \mathrm{m}$ is also in the band. The fact that the $0.5 \mu \mathrm{m}$ wide $L$-prime band centered at 3.7-3.8 $\mu \mathrm{m}$ shows nearly as strong a coronal phase signature as does the $L$ band implies that [Al VI] at $3.66 \mu \mathrm{m}$, common to both bands, is the predominant source of the observed excess. Less pronounced coronal emission phase signatures are seen in the $K$ [Fig. 2(c)], $M$ [Fig. 2(f)], $8.7 \mu \mathrm{m}$ [Fig. 2(g)], and $N$ [Fig. 2(h)] light curves. The filters at
TABLE 3. Speed class of novae plotted in Fig. 4.

\begin{tabular}{lccc}
\hline \hline Nova & Type & $t_{2}$ (days) & $t_{3}$ (days) $^{3}$ \\
\hline V1500 CYg & undetermined & 3 & 6 \\
V1974 CYg & ONeMg & 24 & 44 \\
V1668 CYg & CO & 15 & 30 \\
LW Ser & CO & 34 & 55 \\
NQ Vul & Co & 42 & 64 \\
PW VuI & undetermined & 64 & 100 \\
QU Vu1 & ONeMg & 27 & 40 \\
\hline
\end{tabular}

$$
\begin{aligned}
& \text { 1 Data from Gehrz } 1988 \text { except for v1974 Cyg (data from Hayward } \\
& \text { et al. 1992) } \\
& 2 \text { Time for visible light to decline by three magnitudes } \\
& 3 \text { Time for visual light to decline by three magnitudes } \\
& 4 \text { Formed an optically thin dust shell }
\end{aligned}
$$

wavelengths longer than $10 \mu \mathrm{m}$ are less satisfactory as diagnostics of the IR coronal phase because of contamination by silicate dust emission.

\subsection{The $K$ and L Light Curves of ONeMg (Neon) and CO Novae}

We compare the $K(2.3 \mu \mathrm{m})$ and $L(3.6 \mu \mathrm{m})$ light curves of QU Vul with those of other recent classical novae in Figs. 4(a) and 4(b). V1500 Cyg and PW Vul (Saizar et al. 1991) are shown for comparison as paradigms of the light curve development for $\mathrm{ONeMg}$ and $\mathrm{CO}$ novae, respectively, that do not exhibit persistent IR coronal emission or dust formation phases. V1500 Cyg exhibited a short-lived IR coronal phase (Grasdalen \& Joyce 1976). V1974 Cyg (Hayward et al. 1992; Woodward et al. 1995a) is a recent neon novae that shows neon and IR coronal emission phases similar to those in QU Vul, but which is developing on a somewhat faster time scale. LW Ser and NQ Vul are the prototypical examples of faster $\mathrm{CO}$ novae that formed optically thick (at $V$ ) dust shells. V1668 Cyg was a CO nova that formed an optically thin (at $V$ ) dust shell, but showed a similar light curve structure to the $\mathrm{CO}$ novae that form optically thick dust shells. In comparing the temporal development of novae of various types, it is important to select novae with comparable light decline rates. Some investigators (e.g., Bode \& Evans 1982) have suggested that dust formation may be related to the light decline rate, with novae having slower light decline rates tending to form more dust. Table 3 gives the light decline rates of the novae whose light curves are compared in Fig. 4. It can be seen that there are some interesting exceptions to light decline rate rules. PW Vul, the slowest of the novae described in Table 3, had neither strong dust nor pronounced IR coronal emission. V1668 Cyg declined in intensity more rapidly than either of the neon novae QU Vul or V1974 Cyg, but formed an optically thin dust shell instead.

It is apparent that near-IR light curves are an excellent diagnostic for differentiating $\mathrm{CO}$ and $\mathrm{ONeMg}$ novae. $\mathrm{CO}$ novae that form dust can be distinguished from $\mathrm{ONeMg}$ novae by the strong maximum in the $K$ and $L$ light curves 50 to 100 days after the outburst. This maximum is caused by the thermal reradiation of energy absorbed from the central engine by circumstellar dust grains that nucleate and grow in the ejecta. The subsequent light decline in these novae pre- 
sumably results from the cessation of grain nucleation and growth in the expanding ejecta [see Gehrz et al. (1980a, b)]. ONeMg novae, typified by QU Vul, initially show a rapid exponential decline in all light curves where the cooling is dominated by the thermal bremmstrahlung continuum. Later, when IR coronal emission becomes important, the light curves in bandpasses dominated by emission lines show a strong recovery with respect to the initial decline rate. For example, the $K$ and $L$ light curves, where the cooling is dominated by emission from IR coronal, lines exhibit longlived excesses over the flux levels that would be predicted from the exponential decay rate established prior to the onset of the IR coronal phase. Gehrz (1988a) has pointed out that the thermal continuum emission from dust could mask substantial levels of IR coronal emission during the dust formation phase in DQ Her-type novae. However, a comparison of the $K$ and $L$ light curves of $\mathrm{CO}$ and $\mathrm{ONeMg}$ novae makes it clear that the thermal dust emission phase decays so rapidly that it is unlikely that dust continuum emission could overwhelm the characteristic IR signature of a neon nova with a persistent IR coronal phase like QU Vul for more than a few hundred days following the outburst.

It is especially clear from Fig. 4(b) that the secondary rise in the $L$ light curve is a particularly powerful diagnostic of the onset and duration of the IR coronal phase. We conclude that $L$-band photometry is an excellent tool for studies of novae in other galaxies using sensitive space observatories such as ISO or SIRTF, and note that such photometry can also be useful for initiating spectroscopic studies of novae at critical stages of the outburst development.

Finally, Figs. 4(a) and 4(b) clearly show a peculiar tendency of nova IR light curves to decay exponentially over rather long time scales at some epochs. Data presented by Ciardullo et al. (1990) indicates that such behavior is also characteristic of the $\mathrm{H} \alpha$ and $B$ light curves of V1500 Cyg and several novae in M31. The mean lifetime ( $e$-folding time) for the IR decline rate in a given nova appears to vary from wavelength to wavelength, and the mean life for the light decline at a given wavelength varies from nova to nova as well. For example, the light curves of $\mathrm{CO}$ novae decline exponentially [see Figs. 4(a) and 4(b)] following the peak resulting from the dust formation episode. Figures 4(a) and 4(b) show that the light curves of the ONeMg and other novae show exponential declines at late phases of their temporal evolution as well. In supernovae, this behavior is known to result from the decay of radioactive elements that power the ejecta through gamma ray emission (see Gehrz 1988b). It seems doubtful that the interpretation is so straightforward for novae [e.g., see Pistinner et al.'s (1994) unsuccessful attempt to model conditions under which radioactive decay of $\beta$-unstable nuclei might power the ejecta]. The explanation of this interesting property of nova light decline rates presents a challenge to the theoretical community.

\section{CONCLUSIONS}

We have presented an analysis of 1.25 to $19.5 \mu \mathrm{m}$ broadband IR photometric measurements acquired during the eight year period following the eruption of the prototypical ONeMg "neon nova" QU Vul. Our main conclusions are the following.

(1) The IR SED of QU Vul's ejecta evolved through several phases. An early free-free emission phase was followed by a persistent IR coronal phase in which the free-free continuum had superimposed emission lines from near-IR forbidden atomic transitions of members of the $B$ and $F$ isoelectronic sequences. Emission from [Ne VI] at $7.6 \mu \mathrm{m}$, and [Ne II] at $12.8 \mu \mathrm{m}$ also appeared during the IR coronal phase. The data are consistent with the interpretation that the central engine of the nova system maintained a temperature of nearly $500,000 \mathrm{~K}$ during the period from 1 to $3 \mathrm{yr}$ following the eruption.

(2) Broad emission features at 10 and $20 \mu \mathrm{m}$ were observed about a year after the eruption, suggesting that small amounts of silicate dust condensed in the ejecta.

(3) The IR light curves of QU Vul define the prototypical temporal development scenario for neon novae and show that the temporal development of persistent IR coronal emission phase in ONeMg novae can be traced by broadband $K$, $L$, and $L^{\prime}$ photometry. The most striking signature is produced in the $L$ band, which contains emission from [Mg VIII] at $3.02 \mu \mathrm{m},[\mathrm{Al} \mathrm{VI}]$ at $3.66 \mu \mathrm{m}$, and [Si IX] at $3.92 \mu \mathrm{m}$.

(4) Using data from our previous studies of classical novae, we show how $K$ and $L$ IR light curves can be used to distinguish between $\mathrm{ONeMg}$ novae with persistent IR coronal activity and dust-forming CO novae. $L$ is a particularly useful diagnostic.

(5) Light decline rate is shown to be an ambiguous indicator for predicting the fate of a given nova in terms of whether it will be a $\mathrm{CO}$ or an $\mathrm{ONeMg}$ event. We note a peculiar tendency of the IR light curves of novae to decay exponentially after the initial free-free expansion phase. The mean life of the decay is different for each nova and for each photometric wavelength at which a given nova is observed. Our data offer no obvious explanation for this effect, and we suggest that its explanation is a challenge to nova theorists.

(6) The light curves and SEDs presented here can be used as templates for photometric studies of novae in nearby galaxies using high sensitivity space platforms like ISO and SIRTF. Ground-based IR photometric monitoring of nova light curves and broadband SEDs can also be used to trigger spectroscopic observation programs at appropriate epochs in the temporal development of the outburst.

We thank A. Bentley, D. Dietz, G. Grasdalen, J. Hackwell, C. Jaworoski, D. Mikelson, M. Oswalt, and R. Searna for their participation in some of the observations at the Wyoming Infrared Observatory. Sumner Starrfield, who refereed the original manuscript, offered useful suggestions that refined our representation of the contributions of novae to the ISM and that clarified our assignment of several novae to the $\mathrm{CO}$ and $\mathrm{ONeMg}$ classes. Support for the Wyoming participation in these observations during 1984-1986 was funded through grants to R.D.G. and his collaborators by the National Science Foundation, the United States Air Force, and the University of Wyoming Department of Physics and As- 
tronomy. The Minnesota/Wyoming participation after 1986 was supported by grants to the Minnesota IR Astronomy group by the National Aeronautics and Space Administration, the United States Air Force, the National Science Foundation, and the Graduate School at the University of Minne- sota. The Caltech group is supported by the National Science Foundation and is grateful for the assistance of the Palomar staff in making these observations. C.E.W. was supported by the Ford Foundation while he was a resident at the University of Minnesota during 1990-1991.

\section{REFERENCES}

Benjamin, B., \& Dinerstein, H. L. 1990, AJ, 100, 1588

Bergner, Yu. K., Bondarenko, S. L., Miroshnichenko, A. S., Yudin, R. V., Yutanov, N. Yu., Kuratov, K. S., and Mukanov, D. B. 1984, IAU Circ. No 4033

Bergner, Yu. K., Miroshnichenko, R. V., Yudin, N. Yu., Yutanov, K. G., Dzakusheva, K. G., Ilyin, V. V., Kuratov, K. S., \& Mukanov, D. B. 1988, Ap\&SS, 149, 63

Bode, M. F., \& Evans, A. 1982, MNRAS (UK), 200, 175

Bode, M. F., \& Evans, A. 1989, in Classical Novae, edited by M. F. Bode and A. Evans (Wiley, London), p. 163

Bode, M. F., Evans, A., Whittet, D. C. B., Aitken, D. K., Roche, P. R., \& Whitmore, B. 1984, MNRAS (UK), 207, 897

Bode, M. F., Evans, A., Whittet, D. C. B., Aitken, D. K., Roche, P. R., \& Whitmore, B. 1984, MNRAS (UK), 207, 897

Burton, M., Longmore, A., \& Zinnecker, B. 1984, IAU Circ. No. 4024

Ciardullo, R., Shafter, A. W., Ford, H. C., Neill, J. D., Shara, M. M., \& Tomaney, A. B. 1990, ApJ, 356, 472

Elias, J. H., Frogel, J. A., Matthews, K., \& Neugebauer, G. 1982, 87, 1029 Ennis, D., Becklin, E. E., Beckwith, S., Elias, J., Gatley, I., Matthews, K., Neugebauer, G., \& Willner, S. P. 1977, ApJ, 214, 478

Ferland, G. L., \& Shields, G. A. 1978, ApJ, 224, L15

Gallagher, J. S., \& Ney, E. P. 1976, ApJ, 204, L35

Gehrz, R. D. 1988a, ARA\&A, 26, 377-412

Gehrz, R. D., 1988b, Nature, 333, 705

Gehrz, R. D. 1990, in Physics of Classical Novae, edited by A. Cassatella and R. Viotti (Springer, Berlin), p. 138

Gehrz, R. D. 1995, Abano-Terme Conference on Cataclysmic Variables (Kluwer, Dordrecht) (in press)

Gehrz, R. D., \& Hackwell, J. A. 1978, S\&T, 55, 467

Gehrz, R. D., Grasdalen, G. L., \& Hackwell, J. A. 1985, ApJ, 298, L47, erratum 1986; ApJ, 306, L49 (Paper I)

Gehrz, R. D., Grasdalen, G. L., \& Hackwell, J. A. 1987, Infrared Astronomy, in Encyclopedia of Physical Science \& Technology (Academic, New York), Vol. 2, p. 53

Gehrz, R. D., Hackwell, J. A., \& Jones, T. W. 1974, ApJ, 191, 675

Gehrz, R. D., Truran, J. W., \& Williams, R. E. 1993, in Protostars \& Planets III, edited by E. Levy and J. Lunine (University of Arizona Press, Tucson), p. 75
Gehrz, R. D., Ney, E. P., Grasdalen, G. L., Hackwell, J. A., \& Thronson, H. A. 1984, ApJ, 281, 303

Gehrz, R. D., Grasdalen, G. L., Greenhouse, M., Hackwell, J. A., Hayward, T., \& Bentley, A. F. 1986, ApJ, 308, L63 (Paper II)

Gehrz, R. D., Grasdalen, G. L., Hackwell, J. A., \& Ney, E. 1980a, ApJ, 237, 855

Gehrz, R. D., Hackwell, J. H., Grasdalen, J. A., Ney, E. P., Neugebauer, G., \& Sellgren, K. 1980b, ApJ, 239, 570

Gehrz, R. D., Harrison, T. E., Ney, E. P., Matthews, K., Neugebauer, G., Elias, J., Grasdalen, G. L., \& Hackwell, J. A. 1988, ApJ, 329, 894

Gehrz, R. D., et al. ApJ, 421, 762

Geisel, S. L., Kleinmann, D. E., \& Low, F. J. 1970, ApJ, 161, L101

Grasdalen, G. L., \& Joyce, R. R. 1976, Nature, 259, 187

Greenhouse, M. A., Grasdalen, G. L., Hayward, T. L., Gehrz, R. D., \& Jones, T. J. 1988, AJ, 95, 172

Greenhouse, M. A., Grasdalen, G. L., Woodward, C. E., Benson, J., Gehrz, R. D., Rosenthal, E., \& Skrutskie, M. F. 1990, ApJ, 352, 307

Hayward, T. L., Gehrz, R. D., Miles, J. W., \& Houck, J. R. 1992, ApJ, 401(2), L101-L104, 617

Hyland, A. R., \& Neugebauer, G. 1970, ApJ, 160, L177

Klemola, A. R. 1984, IAU Circ. No. 4024

Lance, C. M., McCall, M. L., \& Uomoto, A. K. 1988, ApJS, 66, 15

Mattei, J. A. 1994, Am. Assoc. Var. Star Obs. (private communication of data)

Neugebauer, G., Oke, J. B., Becklin, E. E., \& Matthews, K. 1979, ApJ 230,

Ney, E. P., \& Hatfield, B. F. 1978, ApJ, 219, L111

Ögelman, H., Krautter, J., \& Beuermann, K. 1987, A\&A, 177, 110

Pistinner, S., Shaviv, G., \& Starrfield, S. 1994, ApJ, 437, 794

Saizar, P., et al., 1991, ApJ, 367, 310

Saizar, P., et al., 1992, ApJ, 398, 651

Williams, P. M., \& Longmore, A. J. 1984, MNRAS (UK), 207, 139

Woodward, C. E., Gehrz, R. D., Jones, T. J., \& Lawrence, G. F. 1992, ApJ, 384(2), L41-L45

Woodward, C. E., et al. 1995a, ApJ, 438, 921

Woodward, C. E., Gehrz, R. D., Jones, T. J., Lawrence, G. R., \& Skrutskie, M. 1995 b, in preparation 